\title{
NOUVELLE MÉtHOdE POUR LE PAIEMENT DU LAIT D'APRÈS SA TENEUR EN MATIËRE GRASSE
}

\author{
par \\ RAPHAEL MOREAU
}

Depuis le décret du 4 septembre 1956, les industriels laitiers effectuent le paiement du lait de vache d'après sa teneur en matière grasse. Celle-ci doit done pouvoir être déterminée de façon aussi exacte que possible pour une période donnée, tout en n'entraînant pour l'industriel acheteur que des frais réduits au minimum. La précision de la détermination est d'autant plus nécessaire que la valeur actuelle du gramme de matière grasse est relativement élevée. Le vendeur, aussi bien que l'acheteur, doivent pouvoir bénéficier de garanties sérieuses afin que les transactions aient toutes les chances de s'effectuer dans un climat, bien souhaitable, de confiance réciproque.

Pourtant, selon les méthodes actuellement utilisées les opérations s'effectuent hors la présence du vendeur et le super-contrôle revêt souvent un caractère purement psychologique. Un doute peut ainsi naître dans l'esprit du fournisseur. Même si les critiques ne sont pas fondées, ce doute peut facilement se transformer en un vif mécontentement lorsque la teneur en matière grasse trouvée n'est pas conforme à celle escomptée.

C'est ainsi qu'il nous a paru intéressant de proposer $\left(^{*}\right)$ une nouvelle méthode nous paraissant donner, tant au vendeur qu'à l'acheteur, des garanties indiscutables. Cette nouvelle méthode permet un super-contrôle à la fois simple et rigoureux, bien que d'un prix de revient très inférieur à celui des méthodes usuelles.

Dans l'espoir d'amener la disparition de pénibles contestations, nous soumettons done à la critique de l'expérience, une réalisation qui nous semble, dès maintenant, valable.

\section{PRINCIPE}

Lors de chaque contrôle, nous prélevons un échantillon delait proportionnel à la quantitélivrée, ce qui est rendu possible par l'utilisation d'une "pipette-sonde" spéciale, robuste et facile à nettoyer, ainsi que d'un récipient standard dans lequel est introduit le lait fourni.

Même si le lait, par suite d'un brassage imparfait, n'est pas absolument homogène, l'échantillon obtenu représente bien la

$\left(^{*}\right)$ Ce transvasement, officiellement prévu par la réglementation sur la Répression des fraudes, peut d'ailleurs ici, être supprimé - ce.qui, sur le plan bactériologique, constituerait un avantage de l'hygiène. Il suffit, en effet, pour que les échantillons prélevés soient toujours proportionnels à la quantité de lait livrée que les livraisons d'un même fournisseur soient effectuées dans des bidons d'un seul modèle. 
qualité moyenne du lait livré, car le prélèvement est opéré sur toute la hauteur du lait transvasé dans le récipient intermédiaire (*).

Les divers prélèvements effectués au cours d'un même mois sont introduits successivement dans le même flacon, conçu à cet effet, et contenant un conservateur. Le flacon reste détenu pendant tout le mois par le fournisseur de lait et toutes dispositions sont prises pour qu'il soit inviolable.

A la fin du mois, les flacons dans lesquels ont été introduits les échantillons successifs sont collectés et déposés au laboratoire d'analyse de la laiterie.

L'ouverture de ces flacons, puis la détermination de la teneur en matière grasse du contenu de chacun d'eux sont ensuite effectués en présence du super-contrôleur mandaté par les agriculteurs.

Une simple multiplication donne alors la valeur de la fourniture du mois.

En cas de contestation, une seconde analyse peut être effectuée par le super-contrôleur lui-même, en présence du représentant qualifié de la laiterie.

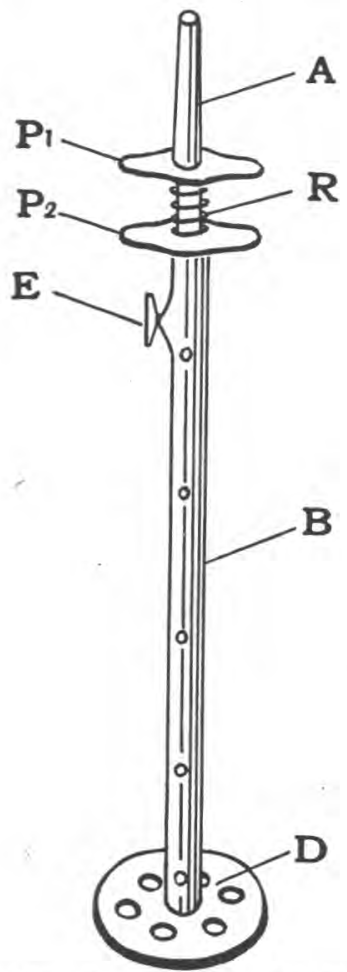

\section{MATÉRIEL}

Le matériel nécessaire comprend : une sonde-pipette, des flacons pour le stockage des échantillons, ainsi que des boîtiers protecteurs et, éventuellement, un récipient intermédiaire.

10 "Sonde-pipette".

Cette sonde-pipette (fig. 1) se compose d'un tube cylindrique $\mathrm{B}$ comportant à sa partie supérieure une poignée $\mathrm{P}^{2}$ et à sa partie inférieure fermée un disque à larges perforations $\mathrm{D}$.

Dans ce tube B est monté, à frottement, un deuxième tube cylindrique $\mathrm{A}$ dont l'extrémité effilée supporte une poignée $\mathrm{P}_{1}$.

Entre $\mathrm{P}_{1}$ et $\mathrm{P}^{2}$ un ressort $\mathrm{R}$ tend à écarter les deux poignées.

Les deux tubes concentriques A et B comportent chacun, suivant deux génératrices opposées, des trouséquidistants (2).

(*) Voir page précédente.

(2) Les trous peuvent être remplacés par des lumières rectangulaires rendant ainsi possible le prélèvement sur toute la hauteur du liquide sans discontinuité. En

FIG. 1. - Sonde-pipette. 
La position de ces trous est telle qu'à la suite d'une pression exercée sur $\mathrm{P}_{1}$, les trous du tube $\mathrm{A}$ viennent coïncider exactement avec ceux du tube B. Dans cette position, un liquide extérieur à la pipette peut donc pénétrer à l'intérieur du tube $\mathrm{A}$ et s'y trouve emprisonné lorsque la pression exercée sur $\mathrm{P}_{1}$ cesse.

En effet, à partir de ce moment, le ressort tend à éloigner $\mathbf{P}_{\mathbf{1}}$ de $\mathrm{P}^{2}$ et entraîne le tube $\mathrm{A}$ solidaire de $\mathrm{P}_{1}$. Les trous homologues des deux tubes ne coïncident plus et il n'y a plus de communication possible entre le milieu extérieur et l'intérieur de la pipette.

Une vis est adaptée sur un ergot $\mathrm{E}$ soudé sur le tube B. Son extrémité se trouve dans une rainure de A et limite ainsi le mouvement de $A$ de manière que les trous de $A$ ne viennent pas coïneider avec des trous de $B$ situés à un niveau supérieur.

Par retournement de la pipette, le liquide isolé dans le tube A s'écoule par le bout verseur et est faeilement recueilli.

Le rapport des diamètres du tube intérieur $\mathrm{A}$ de la pipettesonde et du récipient intermédiaire permet de prélever environ $1 \mathrm{~cm}^{3}$ par litre de lait livré.

\section{Flacons de prélèvements.}

Les échantillons prélevés, sont stockés dans des bocaux du type "Familia Wiss" dont la capacité est, selon l'importance des fournitures et le nombre de prélèvements envisagé au cours d'un mois, de 250,500 ou $1000 \mathrm{~cm}^{3}$. Il s'agit de simples flacons de verre à large ouverture dont la fermeture est assurée par une capsule métallique comportant un joint et un couvercle à vis. Ces flacons étant standard, il est possible de se les procurer dans le commerce à un prix modéré.

\section{$3^{\circ}$ Bô̂tiers protecteurs.}

Les boîtiers sont destinés à assurer d'une part la protection des flacons de prélèvement contre le risque de casse et d'autre part leur inviolabilité.

Il s'agit de boîtes métalliques perforées à double paroi.

Entre les parois A et B, pendant les périodes chaudes de l'année, il est possible de disposer de la mousse que l'on humidifie périodiquement en plongeant le boîtier dans l'eau.

effet, à une lumière située sur une génératrice succède immédiatement la lumière située sur la génératrice opposée. L'obturation est alors réalisée par une rotation de $90^{\circ}$ du tube intérieur par rapport au tube extérieur. Ce dispositif est, évidemment, préférable pour le prélèvement d'échantillons sur de faibles quantités (jusqu'à 3 litres). 
L'eau ainsi absorbée s'évapore peu à peu en provoquant un abaissement de température (Procédé Kreipser pour la conservation des fruits). L'échantillon de lait est ainsi maintenu dans des limites de température acceptables, d'autant plus que l'extérieur du boîtier peut être peint en blanc de façon à réfléchir les rayons lumineux.

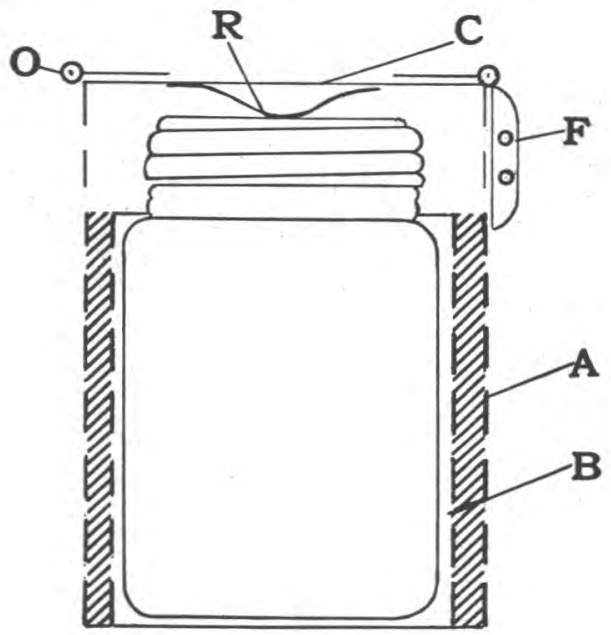

Le couvercle Cest articulé en $\mathrm{O}$ et la fermeture Fest conçue de telle façon qu'elle peut être facilement plombée. Le dispositif de fermeture choisi (loquet et mentonnet) rend pratiquementimpos. sible une rupture accidentelle des plombs.

Enfin, un ressort $R$ immobilise le flacon dans le boîtier lorsque le couvercle est baissé.

FIG. 2. - Boîtier-flacon.

40 Un flacon compte-gouttes, contenant une solution de formaldéhyde à 50 p. 100.

$5^{\circ}$ Un flacon

contenant des comprimés de $0,4 \mathrm{~g}$ de trioxyméthylène.

$6^{\circ}$ Une pince permettant l'apposition de plombs.

70 Récipient intermédiaire.

Ce récipient de forme cylindrique a une capacité d'environ 40 litres. Il est conçu de la même façon que les décalitres courants utilisés dans les laiteries. Son diamètre est approximativement de $36 \mathrm{~cm}$, sa hauteur d'environ $45 \mathrm{~cm}$. Il est gradué en litres.

\section{MODE OPÉRATOIRE}

\section{1. - Identification du fournisseur}

a) Choix de l'indicatif.

Chaque tournée de ramassage est représentée par une lettre de l'alphabet choisie arbitrairement une fois pour toutes. Dans le cadre de chaque tournée un nombre est attribué à chaque four- 
nisseur. L'indicatif d'un fournisseur est donc constitué d'une lettre suivie d'un nombre.

b) Opérations à effectuer.

A chaque fournisseur de lait sont attribués un flacon de prélèvement et un boîtier sur lesquels l'indicatif est reproduit à la peinture afin de rendre toute confusion impossible.

A la fin de chaque mois, le personnel du laboratoire prépare pour le mois suivant les boîtiers et les flacons correspondants et porte l'indicatif $\mathrm{du}$ fournisseur sur la page $\mathrm{du}$ carnet où seront mentionnées les quantités de lait livrées journellement.

Le même personnel établit en même temps, pour chaque tournée de ramassage, un bordereau portant le nom, l'adresse, de chaque fournisseur ainsi que son indicatif. Sur ce bordereau seront ultérieurement enregistrés les résultats des analyses intéressant le mois suivant.

\section{2. - Constitution des échantillons}

Le lait froid (traite de la veille au soir) et le lait chaud (traite du matin) ne peuvent être mélangés. Ils feront donc l'objet de prélèvements distincts, mais ces prélèvements seront ensuite introduits dans le même flacon.

1er temps: Homogénéisation du lait objet du prélèvement.

Le lait d'une même traite contenu dans un ou plusieurs récipients est versé dans le "récipient intermédiaire " ou brassé directement.

Le brassage est réalisé en introduisant la "sonde pipette» et en la soumettant à des mouvements alternatifs de haut en bas et de bas en haut pendant quelques secondes. Le disque perforé situé à la base de celle-ci met le liquide en mouvement et assure une homogénéisation suffisante car le prélèvement est effectué sur toute la hauteur du liquide contenu dans le récipient de transit (1).

\section{$2^{e}$ temps: Prélèvement de l'échantillon.}

La "sonde-pipette» est placée au centre du récipient intermédiaire, le disque reposant sur le fond.

Une pression exercée sur la poignée supérieure met en communication l'extérieur et l'intérieur de la "sonde-pipette». Le tube

(1) L'homogénéisation pourrait, évidemment, être réalisée par tout autre moyen, mais sans doute moins simplement et moins rapidement. 
central se remplit jusqu'au niveau du lait contenu dans le récipient intermédiaire; l'opérateur abandonne alors la pression. Le ressort se détend et entraîne la poignée solidaire du tube central qui remonte. Les trous se trouvent alors obturés et une quantité de lait sensiblement égale à $1 \mathrm{~cm}^{3}$ par litre de lait contenu dans le récipient se trouve emprisonnée dans la sonde-pipette.

Cet échantillon est alors facilement introduit dans le flacon détenu par chaque fournisseur où sont stockés les prélèvements du mois. En effet, par simple retournement de la sonde-pipette, il s'écoule par l'orifice $O$ de la partie effilée du tube intérieur (bout verseur).

Il est évident que si la quantité de lait provenant d'une même traite est supérieure à 40 litres - capacité du récipient intermédiaire - l'opération est répétée autant de fois que cela est nécessaire.

Dans le cas d'une fourniture journalière de quelques litres seulement, lors de chaque contrôle, le prélèvement est répété plusieurs fois, 'mais toujours le même nombre de fois pour un même fournisseur, de manière à ce que le volume prélevé à la fin $\mathrm{du}$ mois puisse permettre au moins deux analyses (environ $25 \mathrm{~cm}^{3}$ ) et reste proportionnel aux quantités livrées.

Lors de chaque contrôle, il faudra avoir soin, avant d'ouvrir le flacon, d'homogénéiser le lait s'y trouvant déjà afin d'éviter toute perte de matière grasse.

$3^{\mathrm{e}}$ temps: Addition du conservateur.

Afin d'assurer la conservation de l'échantillon de lait ainsi obtenu, il sera introduit dans le flacon à prélèvement une solution de formaldéhyde à 50 p. 100 et des comprimés de trioxyméthylène de $0,4 \mathrm{~g}$ en quantité déterminée comme suit :

a) Volumes prélevés compris entre 0 et $100 \mathrm{~cm}^{3}: 2$ gouttes de solution et $1 / 2$ comprimé.

b) Volumes prélevés supérieurs à $100 \mathrm{~cm}^{3}: 2 n$ gouttes de solution $+\frac{n}{2}$ comprimés pour $n$ fois $100 \mathrm{~cm}^{3}$ de lait prélevé, les quantités excédentaires inférieures à $100 \mathrm{~cm}^{3}$ étant comptées pour $100 \mathrm{~cm}^{3}$ lorsqu'elles dépassent $25 \mathrm{~cm}^{3}$.

\section{3. - Stockage des échantillons}

Une fois les opérations déjà décrites exécutées, le flacon qui a reçu le prélèvement et le conservateur est fermé par vissage à fond du couvercle métallique qui, en appuyant sur la capsule, assure une étanchéité parfaite. 
Le flacon est ensuite placé dans le boîtier métallique; le couvercle de celui-ci est rabattu puis immobilisé par un dispositif à loquet et mentonnet prévu à cet effet.

Enfin le boîtier est rendu inviolable par un plombage du dispositif de fermeture, plombage comportant une matrice de repérage.

Le boîtier contenant le flacon de prélèvement est alors accroché à l'anse d'un bidon au moyen d'une chaînette dont l'extrémité est munie d'un mousqueton. Ainsi son transport ne provoque aucune gêne.

Il sera ensuite entreposé à la ferme dans un endroit frais.

A titre de précaution, par temps chaud, le fournisseur aura soin de plonger le boîtier dans l'eau avant de le joindre aux bidons de la livraison qu'il va effectuer.

Dans ces conditions, la mousse se trouvant entre les deux parois cylindriques du boîtier sera humidifiée. Si celui-ci se trouve malencontreusement exposé au soleil, l'évaporation de l'eau, qui s'en suivra, évitera une trop grande élévation de température qui nuirait à la bonne conservation de l'échantillon de lait.

\section{4. - Ramassage des boîtiers}

A la fin du mois, les boîtiers toujours plombés, sont collectés par le personnel de la laiterie. A ce moment, les fournisseurs auront la possibilité d'apposer sur la fermeture un deuxième plomb de garantie comportant une matrice de repérage différente de celle adoptée par la laiterie, et de vérifier que boîtier et carnet portent bien le même indicatif.

Les boîtiers sont ensuite déposés au laboratoire de la laiterie qui avise le super-contrôleur des agriculteurs de la date à laquelle les analyses seront effectuées. Jusqu'à cette date les boîtiers ne devront pas avoir été ouverts.

\section{5. - Opérations effectuées au laboratoire}

Au moment de l'analyse, les boîtiers sont déposés par séries comportant un nombre de boîtiers égal à celui des godets de la centrifugeuse de Gerber.

En présence du super-contrôleur, après avoir vérifié que le plombage est intact, les boîtiers sont ouverts et les flacons renfermant le lait prélevé en sont extraits.

L'analyse est effectuée selon la méthode habituelle de Gerber en prenant les précautions prescrites par l'arrêté du 31 mars 1954 
pour le cas des échantillons de lait formolés : agitation soignée des butyromètres au fur et à mesure de leur remplissage et séjour dans un bain-marie à $65-70^{\circ}$ avant centrifugation.

Les résultats obtenus sont enregistrés sur le bordereau qui, à cet effet, a été établi à la fin du mois précédent.

\section{DISGUSSION DE LA MÉTHODE}

\section{1. - Principales causes d'erreurs inhérentes aux méthodes usuelles}

En examinant les méthodes habituellement employées pour le paiement du lait d'après sa teneur en matière grasse, il apparaît que de nombreuses erreurs peuvent, en toute bonne foi, se produire et fausser les résultats.

Un brassage insuffisant du lait objet du prélèvement, conduit à un échantillon qui ne représente pas le lait moyen. Suivant la partie prélevée l'échantillon peut être tout aussi bien plus riche ou plus pauvre en matière grasse que le lait moyen.

Un bouchage défectueux du flacon de prélèvement peut amener une perte de matière grasse au cours du transport.

Les flacons n'étant pas en général munis de repère individuel, les permutations fortuites sont possibles et même particulièrement faciles si l'ordre de rangement dans les caisses est le seul moyen d'identification. Les caisses elles-mêmes peuvent être par inadvertance, interverties au laboratoire avant l'analyse.

Les calculs nécessaires pour l'obtention de la teneur moyenne en matière grasse d'un lait peuvent également donner lieu à des erreurs, en raison du nombre important d'analyses effectuées.

Des erreurs, lors de l'enregistrement des résultats d'analyses peuvent également se produire, en raison de nombreuses homonymies que dans beaucoup de régions l'on constate parmi les noms patronymiques.

\section{2. - Avantages de la méthode proposée}

\section{A. - Elimination de la plupart des sources d'erreur.}

Les garanties d'exactitude les plus sérieuses sont offertes tant à l'acheteur qu'au vendeur.

a) Aucune confusion entre les flacons de prélèvement n'est possible. L'indicatif du fournisseur est inscrit sur le flacon même, de façon indélébile. Le fournisseur le connaît par la mention portée sur son carnet et peut done constater lui-même, à tout moment, s'il y a ou non une erreur. 
b) L'échantillon soumis à l'analyse représente bien le lait moyen.

En effet, grâce à la " sonde-pipette » le prélèvement est effectué à différents niveaux. Aussi, même si l'homogénéisation n'a pas été complète, l'erreur qui en résulte ne peut être que négligeable, alors que si l'on opère suivant la méthode habituelle, tout manque d'homogénéité se traduit par des taux de matière grasse erronés.

Le tableau I donne les résultats d'un certain nombre d'analyses que nous avons effectuées afin d'apprécier le degré d'exactitude de la méthode que nous proposons.

TABLEAU I

DÉTERMINATION DU TAUX DE MATIĖRE GRASSE D'ÉGHANTILLON DE DIVERS LAITS PRELEVÉS RESPEGTIVEMENT SUIVANT PLUSIEURS TEGHNIQUES

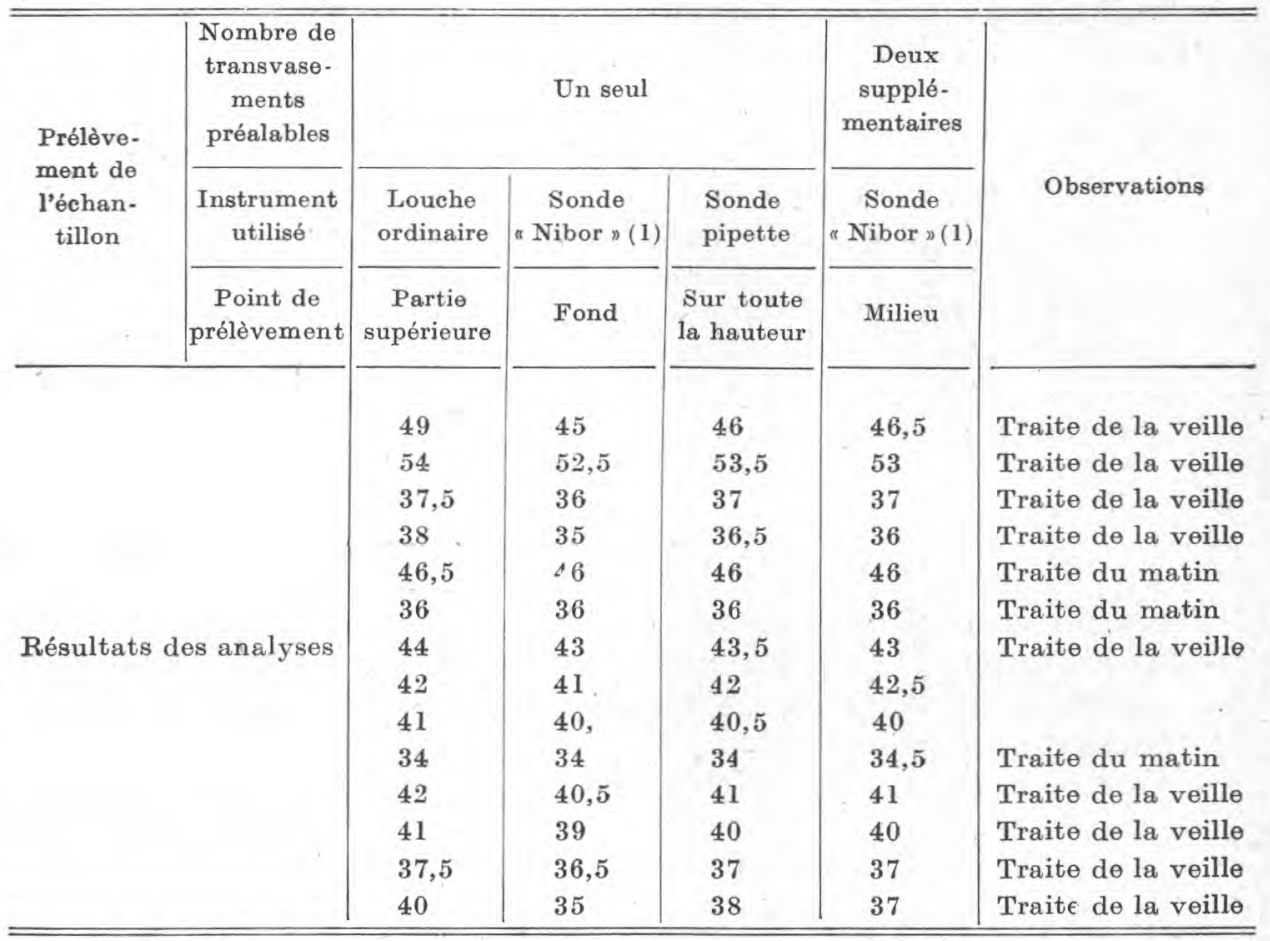

Les résultats sont exprimés en grammes de matière grasse par litre et ont été obtenus en utilisant la méthode de Gerber

(1) Cette sonde, couramment utilisée, est fabriquée et vendue à Surgères (Charente-Maritime) ; elle est constituée par un petit réservoir cylindrique fixé à l'extrémité d'un tube dans lequel passe une tige munie d'une poignée qui commande une soupape d'admission située à la base du réservoir. Elle permet donc de prélever environ $60 \mathrm{ml}$ de lait en un seul point de l'ensemble du liquide. 
Des déterminations de matière grasse ont été effectuées sur le lait transvasé une fois et prélevé d'une part à la partie supérieure, d'autre part, à la partie inférieure de la couche liquide, puis avec la sonde-pipette. Enfin un prélèvement a été effectué sur le lait pouvant être considéré comme parfaitement homogène puisqu'il a subi un brassage prolongé. Les résultats obtenus (tableau I) sont absolument identiques, aux erreurs d'expérience près, pour le lait homogène et le lait prélevé à la sonde-pipette. Par contre, les autres modes de prélèvement ont donné des écarts notables.

Comme on pouvait s'y attendre, lorsque la sonde-pipette n'est pas utilisée, c'est principalement pour le lait provenant de la traite de la veille que des différences sensibles ont été observées dans les résultats des analyses.

Nos essais ont été, évidemment, trop peu nombreux, mais la netteté des résultats permet d'ores et déjà de penser que les conclusions que nous en avons tirées se trouveront confirmées lorsqu'il aura été procédé à de plus amples expérimentations.

c) La simplicité des opérations de prélèvement permet de multiplier ceux-ci au cours d'un même mois sans pour cela augmenter en quoi que ce soit le travail à effectuer au laboratoire et par le service de comptabilité. Il suffit simplement de choisir des flacons et boîtiers de plus grande dimension. On peut ainsi très facilement éliminer les erreurs dues aux variations du taux de matière grasse qui peuvent survenir dans le même mois.

d) Au cas où le fournisseur désirerait faire effectuer une analyse contradictoire, les prélèvements peuvent être effectués en double, sans qu'il s'en suive une perte de temps appréciable sur la route.

e) Le lait prélevé ne peut subir aucune manipulation frauduleuse aussi bien alors qu'il est détenu par le fournisseur que pendant la période qui s'écoule entre l'arrivée au laboratoire et l'analyse. En effet, la fermeture du boîtier est rendue inviolable par un plombage qui peut être double, l'un étant réalisé par le préposé de la laiterie, l'autre par le fournisseur.

D'autre part, fermeture du boîtier et plombage ont été conçus de telle façon que toute rupture accidentelle du plombage est rendue pratiquement impossible. Un climat de confiance est ainsi créé.

f) La conservation de l'échantillon est assurée dans des conditions telles qu'il ne peut pas se produire une altération susceptible d'influencer la teneur en matière grasse du lait.

Nous avons été amené à adopter, comme conservateur, un mélange formol-trioxyméthylène car son pouvoir de bloquer le 
développement des germes semble supérieur à celui des autres conservateurs usuels ainsi que d'ailleurs l'ont montré BEJAMBES, Mocquot et Pauthe (2).

B. - Simplicité d'exécution.

Malgré la rigueur de la méthode, celle-ci est d'exécution très facile.

Le matériel nécessaire est peu important : un récipient de 40 litres et une sonde-pipette. Il n'est donc pas encombrant. Il est facile à nettoyer car la "sonde-pipette» se démonte très facilement. Il suffit d'enlever une simple vis pour libérer les deux pièces la composant.

L'obligation pour le fournisseur de présenter le boîtier lors de chaque livraison de lait ne peut constituer pour lui une gêne notable. En effet, le boîtier n'est pas encombrant et le flacon est bien protégé, si bien qu'aucune précaution spéciale n'est nécessaire pour éviter la casse de celui-ci. Il suffit d'accrocher le boîtier à l'anse d'un bidon au moyen de la chaîne prévue à cet effet.

En ce qui concerne les opérations au laboratoire, elles sont réduites à une seule détermination de matière grasse par fournisseur et par mois. Le lait est prélevé pour l'analyse directement dans le flacon de stockage sans qu'aucune autre manipulation soit nécessaire.

Compte non tenu des majorations accordées pour propreté et non acidité, il ne reste plus à effectuer, pour les services comptables, qu'une multiplication pour déterminer la valeur du lait livré par un agriculteur pendant un mois.

\section{C. - Diminution des frais à supporter par l'industriel.}

La simplification extrême des opérations comptables ainsi que du travail au laboratoire permettent à l'industriel laitier de réaliser des économies très substantielles.

Nous ne saurions mieux mettre en évidence les importantes économies réalisées sur le flaconnage, la manutention, les réactifs et les opérations comptables qu'en prenant un exemple concret.

Soit le cas d'une laiterie ayant 2000 fournisseurs opérant quatre contrôles par mois et une analyse lors de chaque contrôle. Nous noterons que, selon la méthode habituelle, deux flacons en moyenne sont nécessaires par fournisseur (lait froid et lait chaud) pour le prélèvement sur route et un troisième flacon pour la constitution de l'échantillon moyen.

En ce qui concerne la détermination de la teneur moyenne en matière grasse du lait fourni pendant la période considérée, nous admettrons qu'il est tenu compte des quantités livrées lors de 
chaque contrôle. C'est bien le cas dans la méthode proposée puisque les échantillons prélevés sont proportionnels au nombre de litres de lait fournis. La moyenne arithmétique des résultats d'analyse peut en effet conduire à des erreurs non négligeables (3).

L'état comparatif du flaconnage, des réactifs pour l'analyse et des opérations comptables nécessaires pour l'obtention de la teneur en matière grasse moyenne d'une fourniture mensuelle, est donné par le tableau II.

TABLEAU II

ÉTAT COMPARATIF DU FLAGONNAGE, DES RÉACTIFS ET DES OPÉRATIONS COMPTABLES

\begin{tabular}{|c|c|c|c|c|}
\hline & & $\begin{array}{l}\text { Méthode } \\
\text { habituelle }\end{array}$ & $\begin{array}{l}\text { Méthode } \\
\text { proposée }\end{array}$ & Différences \\
\hline \multirow[t]{3}{*}{$\begin{array}{c}\text { A) Verrerie à } \\
\text { laver }\end{array}$} & $\begin{array}{c}\text { Flacons pour prélèvements } \\
\text { sur route } \ldots \ldots \ldots \ldots \ldots\end{array}$ & 16.000 & 2.000 & \\
\hline & $\begin{array}{r}\text { Flacons pour constitution } \\
\text { de l'échantillon moyen } \\
\text { Total } \ldots \ldots \ldots \ldots \ldots\end{array}$ & $\begin{array}{r}8.000 \\
24.000\end{array}$ & $\begin{array}{r}0 \\
2.000\end{array}$ & -22.000 \\
\hline & Butyromètres .......... & 8.000 & 2.000 & -6.000 \\
\hline \multirow[t]{2}{*}{ B) Réactifs } & Acide sulfurique ......... & 80 litres & 20 litres & -60 litres \\
\hline & Alcool isoamylique ..... & c litres & 2 litres & - 6 litres \\
\hline \multirow{4}{*}{$\begin{array}{l}\text { C) Opérations } \\
\text { comptables }\end{array}$} & Nombres à enregistrer ... & 30.000 & 2.000 & -28.000 \\
\hline & Multiplications $\ldots \ldots \ldots \ldots$ & 8.000 & néant & -8.000 \\
\hline & Additions $\ldots \ldots \ldots \ldots$ & 4.000 & - & -4.000 \\
\hline & Divisions $\ldots \ldots \ldots \ldots \ldots$ & 2.000 & - & -2.000 \\
\hline
\end{tabular}

Il ressort de l'examen du tableau I que la méthode proposée permet de réaliser une économie importante.

Cette économie porte, non seulement sur les réactifs, mais surtout sur la main-d'œuvre nécessaire à la préparation et au lavage du matériel de prélèvement et d'analyse ainsi que sur la main-d'œuvre spécialisée chargée des analyses et des travaux de 
comptabilité. Ces derniers se trouvent ramenés à peu de chose. Les fiches individuelles sur lesquelles sont enregistrés les résultats des analyses effectuées lors de chaque contrôle s'avèrent inutiles.

Il est à souligner qu'en suivant la méthode proposée, il y aura dans le cas considéré, 12 fois moins de flacons et 4 fois moins de butyromètres à laver, que toutes les manipulations relatives à la constitution de l'échantillon moyen sont supprimées et que le nombre des déterminations de matière grasse est 4 fois moindre.

En face de ces économies considérables, une seule dépense supplémentaire réside dans l'achat de boîtiers effectué une fois pour toutes.

\section{GONGLUSION}

Nous avons proposé une méthode qui nous paraît simple et rigoureuse pour le paiement du lait d'après sa teneur en matière grasse.

Parce qu'elle est rigoureuse, elle est susceptible de créer un climat de confiance entre le vendeur et l'acheteur.

Sa simplicité entraîne des économies importantes ainsi que l'amélioration des conditions de travail du personnel des laboratoires d'analyse des laiteries, souvent surmené par le nombre fort élevé d'opérations à réaliser.

Enfin, grâce à cette méthode, le super-contrôle devient facile et efficient.

\section{S UMMARY}

A special probe-pipette is introduced allowing at once, rapid mixing of the bulk of milk to be sampled and a selection spread over the whole of the mass, in height. The additional equipment in particular includes a box-bottle to receive selections. The antiseptic recommended : formaldehyde solution (at 50 p. 100) and tablets of $0,4 \mathrm{~g}$ of Trioxymethylene. Analyses thus occur once a month.

\section{BIBLIOGRAPHIE}

[1] C. R. Acad. Agr., no 11, 558. 1959.

[2] M. Bejambes, G. Mocquot et P. Pauthe. Le Lait, 37, 484. 1957.

[3] R. Moreau. Le Lait, 31, 20. 1951. 